\title{
SURJECTIVE ISOMETRIES OF WEIGHTED BERGMAN SPACES
}

\author{
CLINTON J. KOLASKI
}

(Communicated by Paul S. Muhly)

\begin{abstract}
Let $\Omega$ be a bounded, simply connected domain in $C^{n}=R^{2 n}$, let $F \in L^{1}\left(m_{\Omega}\right)$ be positive and continuous on $\Omega$, and let $B_{F}^{p}(\Omega)=L^{p}(F d m) \cap$ $H(\Omega) \quad(0<p<\infty)$ denote the weighted Bergman space over $\Omega$. We characterize those automorphisms $\Phi$ of $\Omega$ such that the map $f \rightarrow g \cdot(f \circ \Phi)$ is a surjective isometry of $B_{F}^{p}(\Omega)$, including an explicit description of $|g|$.
\end{abstract}

\section{INTRODUCTION}

In [2] it was shown that a surjective (linear) isometry of the Bergman space $B^{p}(\Omega)=L^{p}(m) \cap H(\Omega) \quad(0<p<\infty, p \neq 2)$ must have the form

$$
f \rightarrow g \cdot(f \circ \Phi)
$$

where $\Phi$ is an automorphism of the bounded Runge domain $\Omega$, and $g$ is related to $\Phi$ by (1.2).

Conversely, given an automorphism $\Phi$ of $\Omega$, if there is a function $g$ in $B^{p}(\Omega)$ related to $\Phi$ by

$$
\int_{\Omega}(h \circ \Phi)|g|^{p} d m=\int_{\Omega} h d m
$$

for every function $h$ continuous on $\bar{\Omega}=\Omega \cup \partial \Omega$, then (1.1) defines a surjective isometry of $B^{p}(\Omega)$. In (1.2) dm denotes Lebesque measure.

If the domain $\Omega$ is sufficiently nice, e.g., a ball or polydisc, then for every automorphism $\Phi$ there is a corresponding function $g=g_{\Phi}$ in $B^{p}(\Omega)$ which satisfies (1.2). It was conjectured in [2] that this would always be the case if the group of automorphisms acted transitively on $\Omega$.

In this paper we shall characterize those automorphism $\Phi$ of a bounded, simply connected domain $\Omega$ which generate surjective isometries of the weighted Bergman space $B_{F}^{p}(\Omega) \equiv L^{p}(F d m) \cap H(\Omega)$, where the weight function $F \in$ $L^{1}\left(m_{\Omega}\right)$ is assumed only to be positive and continuous on $\Omega$. Moreover, when

Received by the editors May 31, 1988.

1980 Mathematics Subject Classification (1985 Revision). Primary 46E30; Secondary 30H05, $46 \mathrm{~J} 15$.

Key words and phrases. Bergman space, isometry, automorphism. 
the corresponding function $g_{\Phi}$ exists, an explicit description of $\left|g_{\Phi}\right|$ is given. One of the consequences is to answer the above conjecture affirmatively (cf. Remark (ii) and Theorem (2)).

\section{OUR SETTING}

Let $n$ be a fixed positive integer, let $\Omega$ be a bounded domain in $C^{n}=R^{2 n}$, Let $H(\Omega)$ denote the class of holomorphic functions on $\Omega$, let Aut $(\Omega)$ denote the group of automorphisms (i.e., biholomorphic maps) of $\Omega$, and let $m_{\Omega}$ denote Lebesgue measure on $\Omega$ normalized so that $m_{\Omega}(\Omega)=1$. The closure of $\Omega$ in $\mathbf{C}^{n}$ is denoted by $\bar{\Omega}$.

When referring to the space

$$
B_{F}^{p}(\Omega) \equiv L^{p}\left(F d m_{\Omega}\right) \cap H(\Omega)(0<p<\infty)
$$

we shall always assume that the weight function $F$ satisfies

$$
F \in L^{1}\left(m_{\Omega}\right) \text { is POSITIVE and CONTINUOUS on } \Omega \text {, }
$$

and that this space is given the usual metric of $L^{p}\left(F d m_{\Omega}\right)$.

The following short argument shows $B_{F}^{p}(\Omega)$ is a closed subspace of $L^{p}\left(F d m_{\Omega}\right) \quad(0<p<\infty)$.

Let $a \in \Omega$, choose $r>0$ so that the closed ball $a+2 r \bar{B}$ lies in $\Omega$, and let $\delta>0$ denote the minimum of $F$ on this closed ball.

For $f \in B_{F}^{p}(\Omega)$, and for $z$ in the closed ball $a+r \bar{B}$, the subharmonicity of $|f|^{p}$ gives

$$
|f(z)|^{p} \leq c \int_{z+r \bar{B}}|f|^{p} d m \leq \frac{c}{\delta} \int_{z+r \bar{B}}|f|^{p} F d m \leq \frac{c}{\delta} \int_{\Omega}|f|^{p} F d m
$$

where $c$ is a constant independent of $f$.

If $\left\{f_{j}\right\}$ is a Cauchy sequence in $B_{F}^{p}(\Omega)$, then (2.3) implies that $\left\{f_{j}\right\}$ converges uniformly on compact subsets of $\Omega$. Hence, $B_{F}^{p}(\Omega)$ is closed in $L^{p}\left(F d m_{\Omega}\right) \quad(0<p<\infty)$.

\section{SURJECTIVE ISOMETRIES OF $B_{F}^{p}(\Omega)$}

We now turn our attention to determining when an automorphism generates a surjective isometry.

It is easily shown that, given $\Phi \in \operatorname{Aut}(\Omega)$, if there is a function $g$ in $B_{F}^{p}(\Omega)$ $(0<p<\infty)$ related to $\Phi$ by

$$
\int_{\Omega}(h \circ \Phi)|g|^{p} F d m=\int_{\Omega} h F d m
$$

for every function $h$ continuous on $\bar{\Omega}$, then (1.1) defines a surjective isometry of $B_{F}^{p}(\Omega)$. In the cases considered previously $[2,3]$ there was always such a function $g$, but in the present setting this need not be true. Before proving this we shall require a lemma which gives a converse to (3.1). 
Lemma 1. Let $p>0$, and let $\Phi \in \operatorname{Aut}(\Omega)$. If there is a function $g$ in $B_{F}^{p}(\Omega)$ such that the map $f \rightarrow g \cdot(f \circ \Phi)$ is a surjective isometry of $B_{F}^{p}(\Omega)$, then (3.1) holds.

Proof. Let $X$ be the set of all functions continuous on $\bar{\Omega}$ which satisfy (3.1). By our hypothesis $X$ contains all functions for the form $|f|^{p}$, where $f$ is any analytic polynomial. It is clear from (3.1) that $X$ is a linear space which is closed under complex conjugation. We shall show $X$ is an algebra.

If $p=2$, then $(f+h)(\bar{f}+\bar{h})=|f+h|^{2} \in X$, for every pair of analytic polynomials $f$ and $h$. It now follows easily that $f \bar{h} \in X$. Since $X$ is clearly closed under the supremum norm, an application of the Stone-Weierstrass theorem completes the proof of the case $p=2$.

In a similar manner the case $p \neq 2$ follows from [5, Theorem 7.5.3] with $M=$ algebra of analytic polynomials, $A f=f \circ \Phi, d \mu_{1}=F d m_{\Omega}$ and $d \mu_{2}=$ $|g|^{p} F d m_{\Omega}$.

Before we characterize the automorphisms which generate isometries, let us first observe that this class forms a subgroup of $\operatorname{Aut}(\Omega)$.

Proposition 1. The automorphisms of $\Omega$ which generate isometries of $B_{F}^{p}(\Omega)$ $(0<p<\infty)$ form a subgroup of $\operatorname{Aut}(\Omega)$. Moreover, if $\Phi, \Psi \in \operatorname{Aut}(\Omega)$ have (respectively) corresponding functions $g, h \in B_{F}^{p}(\Omega)$, then

(a) $h \cdot(g \circ \Psi)$ corresponds to $\Phi \circ \Psi$ and

(b) $1 /\left(g \circ \Phi^{-1}\right)$ corresponds to $\Phi^{-1}$.

Proof. (a) is obvious. To see (b) define $U$ and $T$ on $B_{F}^{p}(\Omega)$ by

$$
T f=g \cdot(f \circ \Phi) \text { and } U f=\left[1 /\left(g \circ \Phi^{-1}\right)\right]\left(f \circ \Phi^{-1}\right) .
$$

By our hypothesis, $T$ is a surjective isometry of $B_{F}^{p}(\Omega)$, so the range of $T$ is equal to $B_{F}^{p}(\Omega)$. It is easy to show that $U T(f)=f$, for all $f \in B_{F}^{p}(\Omega)$, and it follows that $U=T^{-1}$; i.e., $U$ is a surjective isometry of $B_{F}^{p}(\Omega)$. This proves (b), and the proposition follows.

We are now able to characterize those automorphisms which generate isometries.

Theorem 1. Let $\Omega$ be a bounded, simply connected domain in $\mathbf{C}^{n}$, and let $\Phi \in \operatorname{Aut}(\Omega)$. Let $p>0$. Then there is a function $g$ in $B_{F}^{p}(\Omega)$ such that the map $f \rightarrow g \cdot(f \circ \Phi)$ is a surjective isometry of $B_{F}^{p}(\Omega)$ if and only if

$$
\log (F \circ \Phi / F)
$$

is pluriharmonic in $\Omega$. Moreover, if (3.2) holds, then $g$ is unique (modulo a unimodular constant), is never zero in $\Omega$, and $g$ is related to $\Phi$ by

$$
|g|^{p}=(F \circ \Phi / F) \cdot|J \Phi|^{2}
$$

where $J \Phi$ denotes the complex Jacobian of $\Phi$.

Proof. Let $p>0$, and let $\Phi \in \operatorname{Aut}(\Omega)$. 
Let us first suppose there is a function $g$ in $B_{F}^{p}$ such that the map $f \rightarrow$ $g \cdot(f \circ \Phi)$ defines a surjective isometry of $B_{F}^{p}(\Omega)$. Then, by Lemma $1, \Phi$ and $g$ are related by (3.1). Thus, letting

$$
G=|g|^{p} F /\left(J_{R} \Phi\right)
$$

where $J_{R} \Phi$ denotes the (real) Jacobian of $\Phi$, we find for every continuous function $h$ on $\bar{\Omega}$,

$$
\begin{aligned}
\int_{\Omega} h F d m & =\int_{\Omega}(h \circ \Phi)|g|^{p} F d m \\
& =\int_{\Omega}\left\{\left[h \cdot\left(G \circ \Phi^{-1}\right)\right] \circ \Phi\right\}\left(J_{R} \Phi\right) d m \\
& =\int_{\Omega} h \cdot\left(G \circ \Phi^{-1}\right) d m .
\end{aligned}
$$

Consequently, $F=G \circ \Phi^{-1}$; which with (3.4) shows

$$
(F \circ \Phi) / F=|g|^{p} /\left(J_{R} \Phi\right) \text {. }
$$

Because our isometry is surjective, there is a function $f \in B_{F}^{p}(\Omega)$ such that $1=g \cdot(f \circ \Phi)$. It follows that $g$ is never zero in $\Omega$; thus $|g|^{p}=\left|g^{p}\right|$, where we use the principal branch of the logarithm in defining roots. We recall [5]; there is a holomorphic function $J \Phi$, the complex Jacobian of $\Phi$, which is related to $J_{R} \Phi$ by $J_{R} \Phi=|J \Phi|^{2}$.

We may now write (3.6) in the form

$$
(F \circ \Phi) / F=\left|g^{p} /(J \Phi)^{2}\right| \text {, }
$$

which shows the function $(F \circ \Phi / F)$ is the modulus of a holomorphic function which is never zero in $\Omega$. Thus (3.2) holds.

Conversely, if we assume (3.2) holds, then [5] our assumption that $\Omega$ be simply connected implies there is a function $f$ in $H(\Omega)$ such that $\log (F \circ \Phi / F)=\operatorname{Re}(f)$; thus

$$
F \circ \Phi / F=\exp [\operatorname{Re}(f)] .
$$

If we define

$$
g=(J \Phi)^{2 / p} \exp (f / p)
$$

then $g$ is a holomorphic function on $\Omega$ which (by (3.8)) satisfies (3.3). Moreover,

$$
\int_{\Omega}|g|^{p} F d m=\int_{\Omega}(F \circ \Phi / F)|J \Phi|^{2} F d m=\int_{\Omega} F d m<\infty .
$$

Hence, $g \in B_{F}^{p}(\Omega)$.

Finally an argument similar to (3.10) shows $g$ is related to $\Phi$ by (3.1); therefore, the map $f \rightarrow g \cdot(f \circ \Phi)$ is a surjective isometry of $B_{F}^{p}(\Omega)$. As noted above $g$ is never zero in $\Omega$, and (3.7) must hold. Clearly (3.7) gives (3.3), which then shows $g$ is unique, modulo a unimodular constant. 
Remarks. (i) The "only if" part is true for any bounded domain.

(ii) If $F \equiv 1$, then (3.2) is satisfied with $f \equiv 0$. Thus every $\Phi \in \operatorname{Aut}(\Omega)$ generates a surjective isometry of $B_{F}^{p}(\Omega) \quad(0<p<\infty)$. Moreover, (3.9) shows the corresponding function $g$ is given by $g=(J \Phi)^{2 / p}$. This result is generalized in Theorem (2).

(iii) Note that (3.2) is independent of $p$. Thus, if an automorphism $\Phi$ generates an isometry for one $p>0$, then it generates an isometry for every $p>0$.

(iv) It is clear from (3.2) that it is the algebraic structure of the weight function $F$, not its behavior near the boundary of $\Omega$, that determines which automorphisms generate isometries. As a simple example let $\Omega$ be the unit disc in C. If $F(z)=\left(1-|z|^{4}\right)^{-x} \quad(0<x<1)$, or if $F(z)=\exp \left[-1 /\left(1-|z|^{2}\right)\right]$, then direct computation shows that (3.2) holds if and only if the automorphism is a rotation.

(v) Again let $\Omega$ be the unit disc in C. If $F(z)=\exp \left(z^{2} \bar{z}+z \bar{z}^{2}\right)$, then (3.2) is satisfied only by the identity automorphism.

(vi) If the analytic polynomials are dense in $B_{F}^{p}(\Omega)$, then the proof of Theorem (2.1) in [2] can be modified to show that every surjective isometry of $B_{F}^{p}(\Omega)$ $(p \neq 2)$ is given by $(1.1)$. Question: Is this true when the analytic polynomials are not dense in $B_{F}^{p}(\Omega)$ ?

\section{SurJective isometries of $A^{p, r}(\Omega)$}

The "classical" weighted Bergman spaces have the weight function $F$ expressed as a power of the Bergman kernel. To be more precise, let

$$
F_{r}(z)=\left[B_{\Omega}(z, z)\right]^{-r} \quad(z \in \Omega)
$$

where $B_{\Omega}$ denotes the Bergman kernel for the bounded domain $\Omega$, and $r$ is greater than some negative constant $K(\Omega)$. The space $B_{F_{r}}^{p}(\Omega)$ is usually denoted as $A^{p, r}(\Omega)[1,3,4]$.

If the group $\operatorname{Aut}(\Omega)$ acts transitively on $\Omega$ (which is true, for example, when $\Omega$ is a bounded symmetric domain), then [6],

$$
B_{\Omega}(\Phi(z), \Phi(w)) \cdot(J \Phi)(z) \cdot(\overline{J \Phi})(w)=B_{\Omega}(z, w)
$$

for all $(z, w) \in \Omega \times \Omega$, and for every $\Phi \in \operatorname{Aut}(\Omega)$.

It now follows easily from (4.1) and (4.2) that

$$
\left(F_{r} \circ \Phi\right) / F_{r}=|J \Phi|^{2 r}
$$

for every $\Phi \in \operatorname{Aut}(\Omega)$ which, together with Theorem (1), gives

Theorem 2. If the group $\operatorname{Aut}(\Omega)$ acts transitively on $\Omega$, then every $\Phi \in \operatorname{Aut}(\Omega)$ generates a surjective isometry of $A^{p, r}(\Omega)$ for all $p>0$ and all $r>K(\Omega)$. Moreover, the corresponding function $g$ is given by

$$
g=(J \Phi)^{(2 r+2) / p}
$$


Remark. (vii) If $\Omega$ is taken to be the unit ball in $\mathbf{C}^{n}$, and if $\Phi \in \operatorname{Aut}(\Omega)$, then [5],

$$
(J \Phi)(z)=\left[\left(1-|a|^{2}\right) /(1-\langle z, a\rangle)^{2}\right]^{(n+1) / 2}
$$

where $\Phi(a)=0$. Thus, Theorem 2 contains Theorem 4 of [3] as a special case (recall there $x=(n+1) r)$.

\section{REFERENCES}

1. C. Horowitz, Zeros of functions in the Bergman spaces, Duke Math. J. 41 (1974), 693-710.

2. C. Kolaski, Isometries of Bergman spaces over bounded Runge domains, Canad. J. Math. 33 (1981), 1157-1164.

3. __ Isometries of Weighted Bergman spaces, Canad. J. Math. 34 No. 4 (1982), 910-915.

4. D. H. Luecking, Representation and duality in weighted spaces of analytic functions, Indiana Univ. Math. J. 34, No. 2 (1985), 319-336.

5. W. Rudin, Function theory in the unit ball of $\mathbf{C}^{n}$, Springer-Verlag, New York, 1980.

6. A. Selberg, Automorphic functions and integral operators, Seminars on Analytic Functions, II, Institute for Advanced Study, Princeton, N.J., 1957, pp. 152-161.

Department of Mathematics, University of Minnesota-Duluth, Duluth, Minnesota 55812 